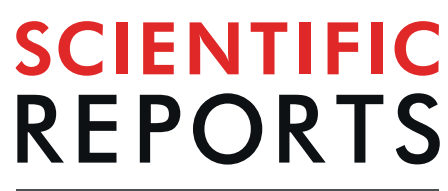

natureresearch

Check for updates

\title{
Structural characterization of
} SnO nanoparticles synthesized by the hydrothermal and microwave routes

\author{
J. S. Dias, F. R. M. Batista, R. Bacani ${ }^{10}$ \& E. R. Triboni ${ }^{\circledR}$
}

SnO particles were synthesized by an alkali-assisted hydrothermal and microwave methods. The aqueous-based reactions were carried out at $\mathrm{pH} \sim 8$, under inert atmosphere (Ar). The reactions were taken under different times, and a full XRD structural analysis was made to evaluate the conversion from the $\mathrm{Sn}_{6} \mathrm{O}_{4}(\mathrm{OH})_{4}$ intermediate to $\mathrm{SnO}$ particles. Williamson-Hall analysis showed that the size and strain of the SnO particles were time and route treatment dependent. Microwave heating yielded a single tetragonal SnO phase after $1 \mathrm{~h}$ of thermal treatment, and TEM images revealed sphericalshaped SnO nanoparticles with an average size of $9(1) \mathrm{nm}$. While by the hydrothermal treatment single SnO phase was obtained only after 4 hours, yielding non-uniform and elongated particles with submicrometric size. A dissolution-recrystallization process was taken into account as the mechanism for $\mathrm{SnO}$ particles formation, in which hydroxylated complexes, $\mathrm{Sn}_{2}(\mathrm{OH})_{6}{ }^{-2}$, then condense to form the oxide. The time-shorting reaction provided by the microwave-assisted synthesis may be attributed to better heat distribution.

Tin monoxide $\left(\mathrm{SnO}-\mathrm{Sn}^{+2}\right)$ and tin dioxide $\left(\mathrm{SnO}_{2}-\mathrm{Sn}^{+4}\right)$ are well-known semiconductors with p-type and n-type electronic properties, respectively ${ }^{1-4}$. Their applications have encompassed technological areas such as optoelectronics, energy storage and sensing ${ }^{5-8}$. $\mathrm{SnO}_{2}$ is a pale-yellow solid with rutile-type structure and wide-bandgap $(E g=3.6 \mathrm{eV})$ and very used for transparent conductive electrodes ${ }^{7}$, gas sensors ${ }^{1,8}$, electrochromic devices ${ }^{3}$ and photoelectrodes $^{3,4}$. While $\mathrm{SnO}$ is a dark solid with tetragonal structure and variable optical bandgap $(E g=2.7-$ $3.4 \mathrm{eV})^{9-11}$, and used for gas sensor devices ${ }^{12}$, electrodes for rechargeable Li-ion batteries ${ }^{13-15}$, supercapacitors ${ }^{16}$, native $\mathrm{p}$-type conducting material ${ }^{17}$, as well as catalyst and photocatalyst ${ }^{18,19}$.

The preparation of the $\mathrm{SnO}$ phase is harder than $\mathrm{SnO}_{2}$ one, due to the favorable oxidation of the $\mathrm{Sn}^{+2}$ to the thermodynamically more stable $\mathrm{Sn}^{+4}$. Therefore, the synthetic challenge is to avoid this favorable oxidation, aside from the implementation of procedures that lead to well-controlled size and morphology. In this context, microand nano-crystalline SnO particles have been made by chemical/physical procedures, such as hydrothermal and solvothermal preparations, electrochemistry, ultrasound- and microwave-assisted routes, ionic liquids ${ }^{19-26}$, and thermal vapor deposition, thermal evaporation ${ }^{3}$, thermal chemical vapor deposition (CVD) ${ }^{4}$, and mechanical ball milling 8 . Moreover, organic additives, surfactants, inert atmosphere $\left(\mathrm{Ar}\right.$ or $\left.\mathrm{N}_{2}\right)$ and reductive $\left(\mathrm{H}_{2}\right)$ gases are applied with purposes of improving the yield forward the lower valence phase $\mathrm{e}^{15,25,27,28}$.

Thermal vapor deposition and a long time of a hydrothermal treatment have been pointed out as the better conditions to obtain single-crystalline $\mathrm{SnO}$ particles ${ }^{26,29}$. Furthermore, in aqueous based-synthesis, the intermediate tin (II) oxyhydroxide, $\mathrm{Sn}_{6} \mathrm{O}_{4}(\mathrm{OH})_{4}$, is often prepared from the hydroxylation of $\mathrm{SnCl}_{2} \cdot 2 \mathrm{H}_{2} \mathrm{O}$ then condensation is taken on elevated temperatures and pressures, in alkaline solutions, or suspensions, to ensure due to speciation for $\mathrm{SnO}$ formation ${ }^{30,31}$.

Herein, conventional- and microwave-based hydrothermal treatments were used to form SnO nanoparticles (SnO-NPs), under inert atmosphere and relatively lower $\mathrm{pH}(\mathrm{pH} \sim 8)$. The synthesis efficiency was compared in terms of reaction time, size and morphological aspects. Microwave heating yielded SnO-NPs with a spherical shape, small average size, and high uniformity upon 2 hours of thermal treatment. Conversely, hydrothermal preparation provided elongated sub-micrometric SnO-NPs with different sizes and morphologies upon 4 hours of 


\begin{tabular}{|c|c|c|c|c|c|}
\hline $\begin{array}{l}\text { Hydrothermal } \\
\text { processing }\end{array}$ & Sample name & $\begin{array}{l}\mathrm{pH} \text { of the } \\
\text { solution }\end{array}$ & Time & $\begin{array}{l}\text { powder } \\
\text { pictures }\end{array}$ & Color \\
\hline \multirow{6}{*}{ 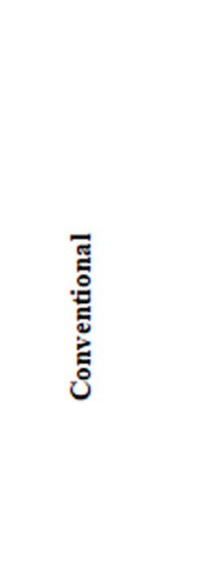 } & HOh 45 & 8.2 & $45 \mathrm{~min}$ & & white \\
\hline & H1h00 & 8.2 & $1 \mathrm{~h}$ & & white \\
\hline & $\mathrm{H} 1 \mathrm{~h} 30$ & 8.2 & $1 \mathrm{~h} 30$ & & black \\
\hline & $\mathrm{H} 2 \mathrm{~h} 30$ & 8.2 & $2 \mathrm{~h} 30$ & & black \\
\hline & $\mathrm{H} 4 \mathrm{~h} 00$ & 8.3 & $4 \mathrm{~h}$ & & black \\
\hline & $\mathrm{H} 6 \mathrm{~h} 00$ & 8.3 & $6 \mathrm{~h}$ & & black \\
\hline \multirow{4}{*}{ 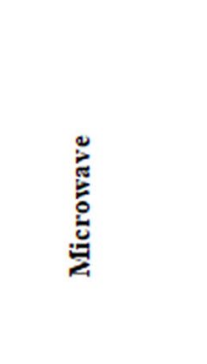 } & $\mathrm{MOh} 45$ & 8.1 & $45 \mathrm{~min}$ & & gray \\
\hline & M1h00 & 8.2 & $1 \mathrm{~h}$ & & dark gray \\
\hline & $\mathrm{M} 1 \mathrm{~h} 30$ & 8.1 & $1 \mathrm{~h} 30$ & & dark gray \\
\hline & $\mathrm{M} 2 \mathrm{~h} 30$ & 8.1 & $2 \mathrm{~h} 30$ & & black \\
\hline
\end{tabular}

Figure 1. Powders obtained under various reaction times; sample names and characteristics.

treatment. The evaluation of the crystalline structures, from $\mathrm{Sn}_{6} \mathrm{O}_{4}(\mathrm{OH})_{4}$ to $\mathrm{SnO}$, in the different reaction times, was determined using $\mathrm{X}$-ray diffraction microstructural analysis and transmission electronic microscopy (TEM) images.

\section{Experimental}

Materials. Tin chloride dihydrate $\left(\mathrm{SnCl}_{2} \cdot 2 \mathrm{H}_{2} \mathrm{O}\right)$, ammonium hydroxide $\left(\mathrm{NH}_{4} \mathrm{OH}\right)$ and hydrochloric acid $(\mathrm{HCl})$ were purchased from Synth, were used without further purification. The water used was double distilled and deionized.

Synthesis of the tin (II) oxyhydroxide $\left(\mathrm{Sn}_{6} \mathrm{O}_{4}(\mathrm{OH})_{4}\right)$ precursor (1). As a general procedure tin chloride dihydrate $\left(0.5 \mathrm{~mol}, \mathrm{SnCl}_{2} .2 \mathrm{H}_{2} \mathrm{O}\right)$ was dissolved in $100 \mathrm{~mL}$ of $0.5 \mathrm{M} \mathrm{HCl}$ solution $(\mathrm{pH} \sim 2)$ which was then transferred to a $300 \mathrm{~mL}$ three-necked round bottom flask and kept under stirring at room temperature and inert atmosphere (Ar). Then, an $\mathrm{NH}_{4} \mathrm{OH}$ solution $28-30 \%(\sim 25 \mathrm{~mL})$ was added dropwise into the acidic solution until $\mathrm{pH} \sim 8$, producing a white cloudy suspension $(\sim 125 \mathrm{~mL})$ of the precursor $\mathrm{Sn}_{6} \mathrm{O}_{4}(\mathrm{OH})_{4}, \mathbf{1}$.

Microwave treatment. A Titan MPS microwave extraction and digestion system (PerkinElmer) with $2.45 \mathrm{GHz}$ magnetrons that provide up to $1,500 \mathrm{~W}$ of power supply. The equipment was set up with a ramp at the temperature of $80^{\circ} \mathrm{C}$, which was reached after $10 \mathrm{~min}$ with a maximum pressure control of $35 \mathrm{bar}$ and an average of $20 \%$ output power. Reactions were carried out into Teflon autoclave reactors which were loaded with $30 \mathrm{~mL}$ from 1, then heated and left for $45 \mathrm{~min}, 1 \mathrm{~h}, 1 \mathrm{~h} 30 \mathrm{~min}$, and $2 \mathrm{~h} 30 \mathrm{~min}$ (sample names showed in Fig. 1). Previously, all loaded-autoclaves were purged with argon for $10 \mathrm{~min}$ to maintain an oxygen-free medium. At the end of each reaction, the vessels were left to chill until room temperature. The powders were filtered off and washed several times with deionized water and dried in a muffle oven in $60^{\circ} \mathrm{C}$ before characterization.

Hydrothermal treatment. For each reaction, $30 \mathrm{~mL}$ of the suspension 1 was put into hydrothermal autoclave reactor with Teflon chamber and also purged with argon to obtain an inert medium, then placed in an muffle oven already pre-heated at $100^{\circ} \mathrm{C}$, for $45 \mathrm{~min}, 1 \mathrm{~h}, 1 \mathrm{~h} 30$ and $2 \mathrm{~h} 30,4 \mathrm{~h}$, and $6 \mathrm{~h}$ (sample names showed in Table 1). After cooling until room temperature, all samples were filtered off and washed several times with distilled water and dried in a muffle oven in $60^{\circ} \mathrm{C}$ before characterization. 


\begin{tabular}{|l|l|l|l|l|l|l|l|l|}
\hline \multirow{2}{*}{ Samples } & $\mathbf{a} / \mathbf{b}_{\mathrm{T}}$ & $\mathbf{c}_{\mathrm{T}}$ & $\mathbf{V}_{\mathrm{T}}$ & $\mathbf{f}_{\mathrm{T}}$ & $\mathbf{a} / \mathbf{b}_{\mathrm{H}}$ & $\mathbf{c}_{\mathrm{H}}$ & $\mathbf{V}_{\mathrm{H}}$ & $\mathbf{f}_{\mathrm{H}}$ \\
\cline { 2 - 9 } & $(\AA)$ & $(\AA)$ & $\left(\AA^{3}\right)$ & $\%$ & $(\AA)$ & $(\AA)$ & $\left(\AA^{3}\right)$ & $\%$ \\
\hline H0h45 & - & - & - & - & $7.9359(7)$ & $9.112(1)$ & $573.86(5)$ & $100(1)$ \\
\hline H1h00 & - & - & - & - & $7.9366(7)$ & $9.112(3)$ & $573.95(4)$ & $100(1)$ \\
\hline H2h00 & $3.8024(3)$ & $4.8353(4)$ & $69.909(3)$ & $87.03(3)$ & $7.950(3)$ & $9.140(5)$ & $577.62(8)$ & $13(1)$ \\
\hline H4h00 & $3.8015(7)$ & $4.8349(9)$ & $69.872(2)$ & $100(2)$ & - & - & - & - \\
\hline H6h00 & $3.8021(6)$ & $4.8351(8)$ & $69.895(2)$ & $100(1)$ & - & - & - & - \\
\hline M0h45 & $3.8012(4)$ & $4.8331(5)$ & $69.837(13)$ & $53(1)$ & $7.9399(9)$ & $9.1225(12)$ & $575.11(12)$ & $47(1)$ \\
\hline M1h00 & $3.8031(10)$ & $4.8366(13)$ & $69.951(3)$ & $100(1)$ & - & - & - & - \\
\hline M1h30 & $3.8029(18)$ & $4.8358(21)$ & $69.936(6)$ & $100.0(8)$ & - & - & - & - \\
\hline M2h30 & $3.8007(3)$ & $4.8346(3)$ & $69.838(8)$ & $100.0(8)$ & - & - & - & - \\
\hline
\end{tabular}

Table 1. Structural paramenters from Rietveld analysis: lattice parameters $(\mathrm{a} / \mathrm{b}, \mathrm{c})_{\mathrm{T}, \mathrm{H}}$, unit cell volume $\left(\mathrm{V}_{\mathrm{T}, \mathrm{H}}\right)$, phase weight fraction $\left(\mathrm{f}_{\mathrm{T}, \mathrm{H}}\right)$, where $\mathrm{T}=$ tin oxide $(\mathrm{SnO}), \mathrm{H}=\operatorname{tin}(\mathrm{II})$ oxyhydroxide $\left(\mathrm{Sn}_{6} \mathrm{O}_{4}(\mathrm{OH})_{4}\right)$. Deviations presented between (), i.e., $3.5 \pm 0.5 \mathrm{~nm}$ means $3.5(5) \mathrm{nm}$.

Nanoparticles characterization. The structural characterization was performed with X-ray diffraction (XRD) measurements of the dried powder samples using a PANalytical Empyrean equipment, with a copper tube $\left(\lambda=1.5418 \AA\right.$ ), Ni filter, and PIXcel ${ }^{3 \mathrm{D}}$ detector, operating at $40 \mathrm{kV}$ and $30 \mathrm{~mA}$, with $2 \theta$ from $10^{\circ}$ to $90^{\circ}$ with a $0.02^{\circ}$ step and counting times of 3 to $5 \mathrm{~s} / \mathrm{step}$. To quantify phases and obtain structural parameters, the Rietveld's powder structure refinement analysis was performed ${ }^{32-35}$ using Fullprof ${ }^{36,37}$ and X'Pert Highscore Plus (PANalytical) ${ }^{38}$ software. The peak shape was assumed as a pseudo-Voigt function with axial asymmetry, preferential direction of (00l) planes, and the background of each pattern was fitted with a polynomial function of degree 5 . Isotropic thermal vibrations were considered. Experimental parameters such as the sample's displacement and absorption were also variables. The method takes a least-square routine to minimize the difference between the observed and simulated powder diffraction patterns with the $\mathrm{R}$ factors, goodness of fit $\left(\mathrm{S}_{\mathrm{GoF}}\right)$ and $\chi^{235}$. The crystallite size and strain were calculated via the Williamson-Hall plot $^{39,40}$.

Morphological characterization was performed with transmission electron microscopy (TEM) images, carried out in a JEOL JEM 2100 with $\mathrm{LaB}_{6}$ filament, $0.23 \mathrm{~nm}$ point resolution and high angular dark field detector (HAADF). Samples were suspended in ethanol and sonicated for 1 hour before measurement. Images were treated with ImageJ software ${ }^{41,42}$.

\section{Results and Discussion}

Structural and morphological characterization. The powders obtained under various reaction times for both conventional and microwave hydrothermal preparations are illustrated in Fig. 1.

XRD results and Rietveld analysis are presented in Figs. 2 and 3, and Tables 1 and 2 respectively. All samples were indexed with the Inorganic Crystal Structure Database (ICSD, FIZ Karlsruhe) database ${ }^{43,44}$.

Tables 1 and 2 present the structural parameters and fraction of the phases, besides the factors of agreement of the adjustment. Since some samples presented more than one crystallographic phase, preferential high direction, and asymmetry of peaks, especially for samples synthesized via conventional hydrothermal thermal treatment, the convergence values such as goodness of fit $\left(\mathrm{S}_{\mathrm{GoF}}\right)$ and global $\chi^{2}$, are relatively higher than expected in general.

Quantification results show that for the conventional hydrothermal method, the complete formation from the tin (II) oxyhydroxide phase, $\mathrm{Sn}_{6} \mathrm{O}_{4}(\mathrm{OH})_{4}\left(\mathrm{P} \overline{4} 2_{1} \mathrm{c}, \mathrm{PDF} \# 01-084-2157\right)^{43}$ to pure tetragonal phase SnO (P4/nmmS, PDF \#01-085-0423 $)^{44}$ happens with at least 4 hours of thermal treatment. Within 2 hours of treatment, it can be observed a large intensity change for (001) and (002) planes, suggesting the formation of rods with growth in the $c$ parameter direction. Lattice parameter and cell volume are similar to those to the standard crystallographic $\mathrm{SnO}$ data and a slight increase with treatment time from 4 to 6 hours (Table 1). For the microwave method, there is already $\sim 50 \%$ formation of $\mathrm{SnO}$ and $\sim 50 \%$ of $\mathrm{Sn}_{6} \mathrm{O}_{4}(\mathrm{OH})_{4}$ phases with $45 \mathrm{~min}$ of treatment. The complete formation of crystalline single-phase $\mathrm{SnO}$ occurs after a 1-hour thermal treatment.

Figure 3 shows TEM images from SnO nanoparticles synthesized by both methods. It can be seen that microwave-assisted synthesis leads to uniformly sized spheres, Fig. 4(a-c), while in the conventional hydrothermal treatment there are agglomerated particles without definite shape, Fig. 4(d-f). The conventional hydrothermal synthesis also produces elongated particles that could be responsible for the large intensity of (001) peaks. The average nanoparticle size for M1h00 is 9(1) nm and, for M2h00 is 4.0(7) nm. For the H6h00 sample, the sizes vary from 10 to larger than $50 \mathrm{~nm}$, the elongated particles have an average length of $30(6) \mathrm{nm}$. However, the microwave treatment leads to a more uniform formation of $\mathrm{SnO}$ nanoparticles instead of the conventional heating method.

Microstructural analysis was performed in order to evaluate the average characteristics of the SnO powders. The Williamson-Hall plot (WHP) is shown in Fig. 5, and the results are displayed in Table 3. This analysis takes into account the crystallite size and the lattice deformations instead of the Scherrer formula that only evaluates the crystallite size $\mathrm{u}^{40,41,45,46}$.

The crystallite size calculated is greater than TEM images mostly because of the agglomerated particles between each other (Table 1), producing an effect on the intensity distribution, since crystal sizes smaller than $100 \mathrm{~nm}$ are harder to analyze via XRD size-strain analysis ${ }^{40,45,46}$. These particle clusters can be seen in Fig. 3(c-f). The crystallite size grows with the conventional hydrothermal treatment time, while in the microwave treatment the crystallite and nanoparticle size decreases. 


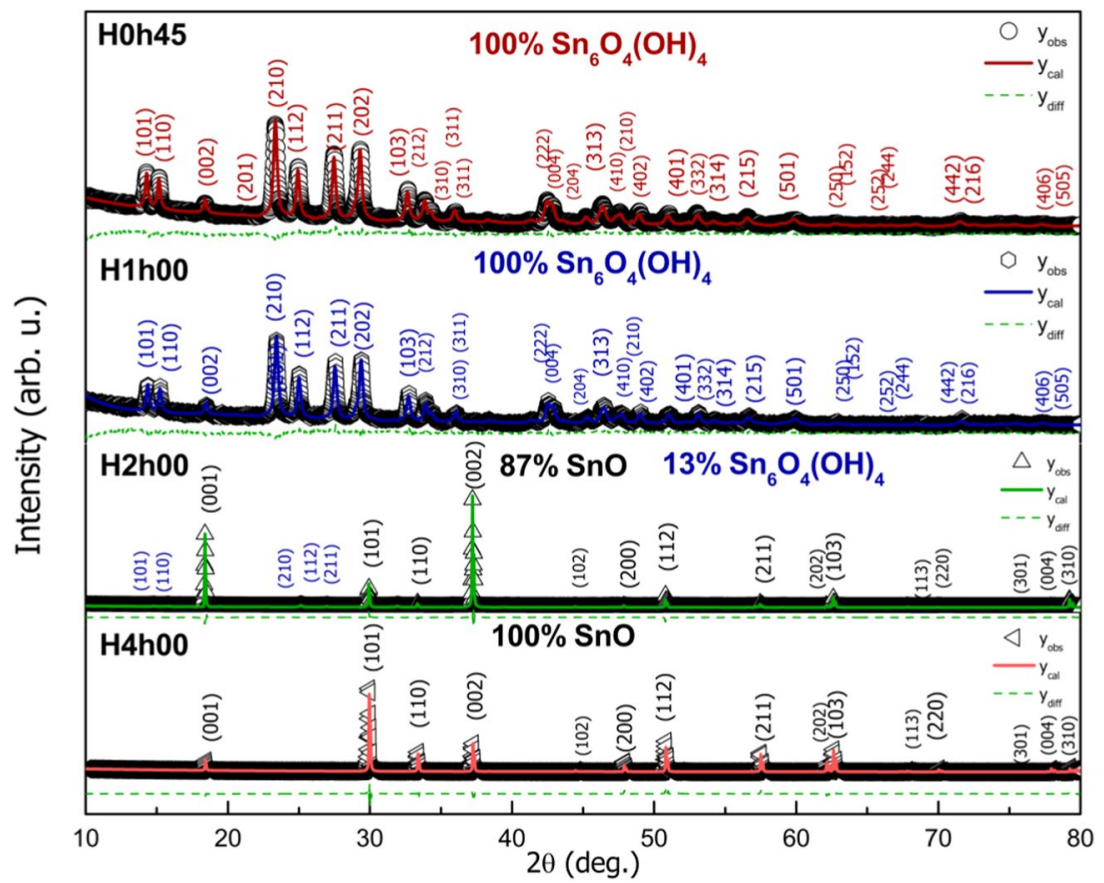

Figure 2. X-ray diffractograms from conventional hydrothermal (H) SnO nanoparticles. The data for H6h00 is similar for H4h00, so it was not presented in this graph. Symbols represent experimental data, the lines are for Rietveld method calculated intensity, and dashed lines denote the difference between experimental and calculated intensities.

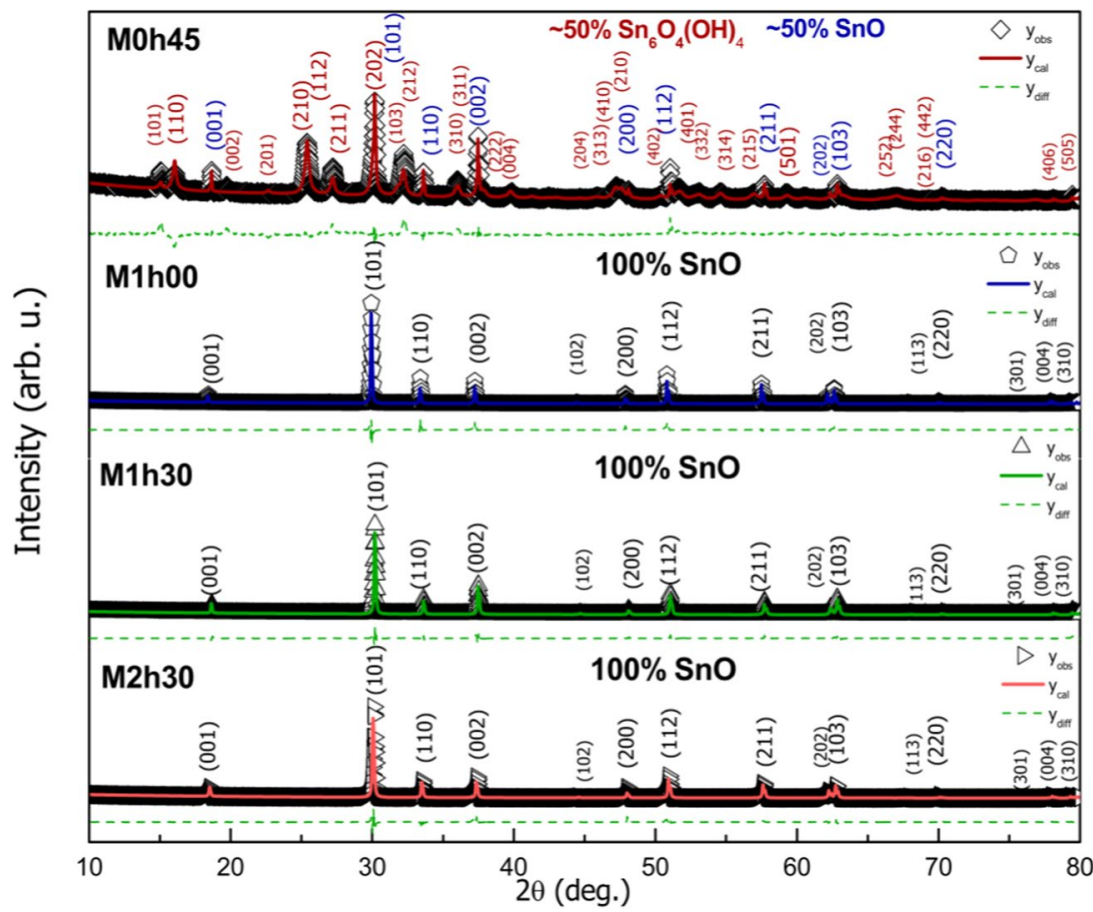

Figure 3. X-ray diffractograms from microwave treatment (M) SnO nanoparticles. Symbols represent experimental data, the lines are for Rietveld method calculated intensity, and dashed lines denote the difference between experimental and calculated intensities.

The linear behavior of data in the WHP is consistent with and regular distribution of crystallite sizes. In this case, for the conventional hydrothermal treatment, the negative strain can be related to particle inhomogeneities, since the strain decreases as de particle size increases and the lattice parameters increase (Table 2). For the 


\begin{tabular}{|l|l|l|l|l|}
\hline Samples & $\mathbf{R}_{\mathrm{WP}}(\%)$ & $\mathbf{R}_{\mathrm{EXP}}(\%)$ & $\mathbf{S}_{\mathrm{GOF}}$ & $\chi^{\mathbf{2}}$ \\
\hline H0h45 & 6.87 & 4.69 & 2.1 & 3.19 \\
\hline H1h00 & 6.78 & 5.07 & 1.8 & 3.21 \\
\hline H2h00 & 16.12 & 4.38 & 3.6 & 6.00 \\
\hline H4h00 & 7.98 & 5.21 & 2.1 & 4.53 \\
\hline H6h00 & 7.74 & 5.02 & 2.1 & 4.44 \\
\hline M0h45 & 8.47 & 4.94 & 3.0 & 5.20 \\
\hline M1h00 & 8.50 & 5.04 & 3.2 & 5.62 \\
\hline M1h30 & 7.71 & 5.10 & 2.1 & 4.61 \\
\hline M2h30 & 7.68 & 5.08 & 2.3 & 5.14 \\
\hline
\end{tabular}

Table 2. Convergence factors of the Rietveld Method $\left(\mathrm{R}_{\mathrm{WP}}, \mathrm{R}_{\mathrm{EXP}}\right)$, Goodness of Fit $\left(\mathrm{S}_{\mathrm{GoF}}\right)$ and global $\chi^{2}$ of the fitting.

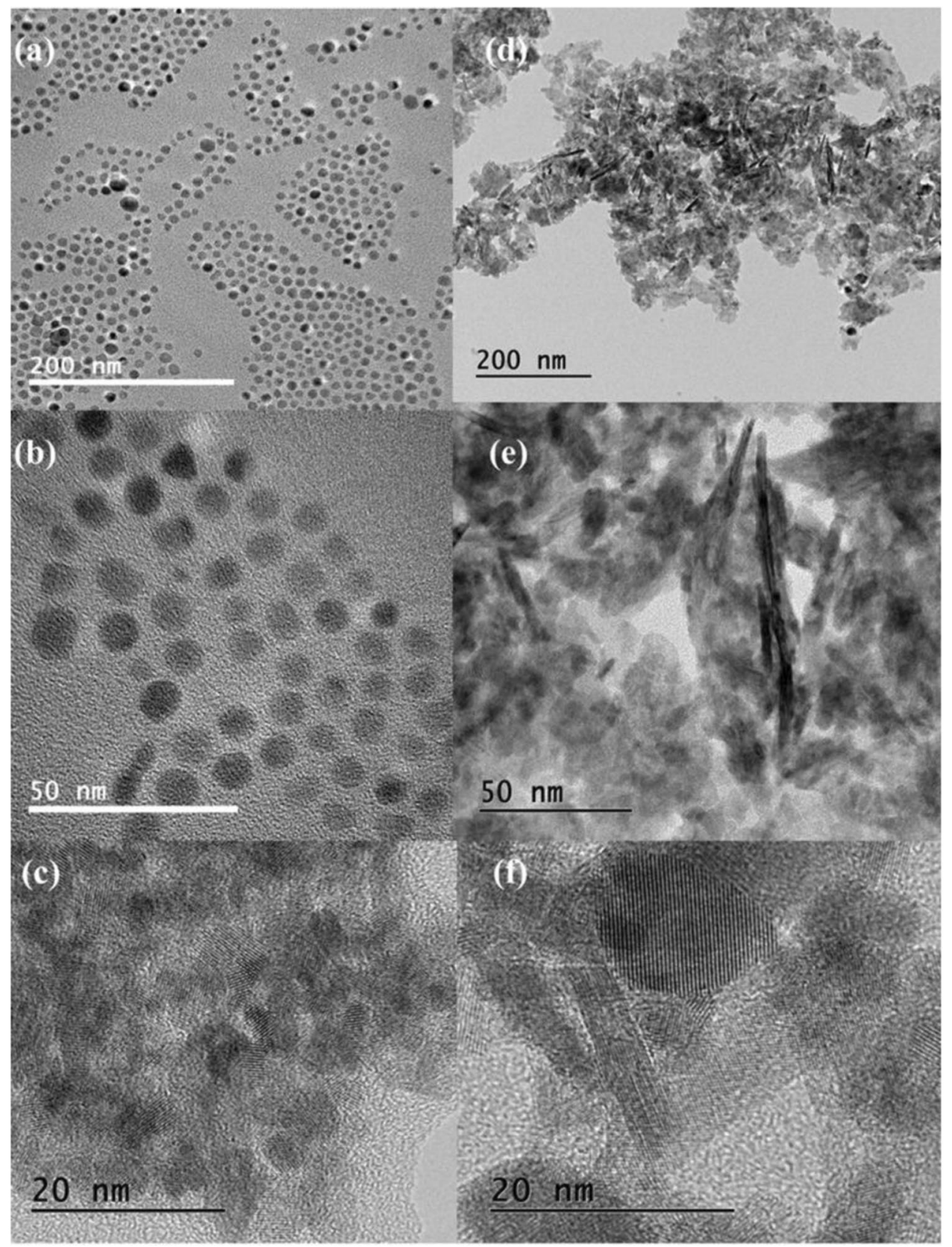

Figure 4. TEM images for SnO nanoparticles. (a) M2h00; (b,c) M1h00; and (d-f) H6h00. 


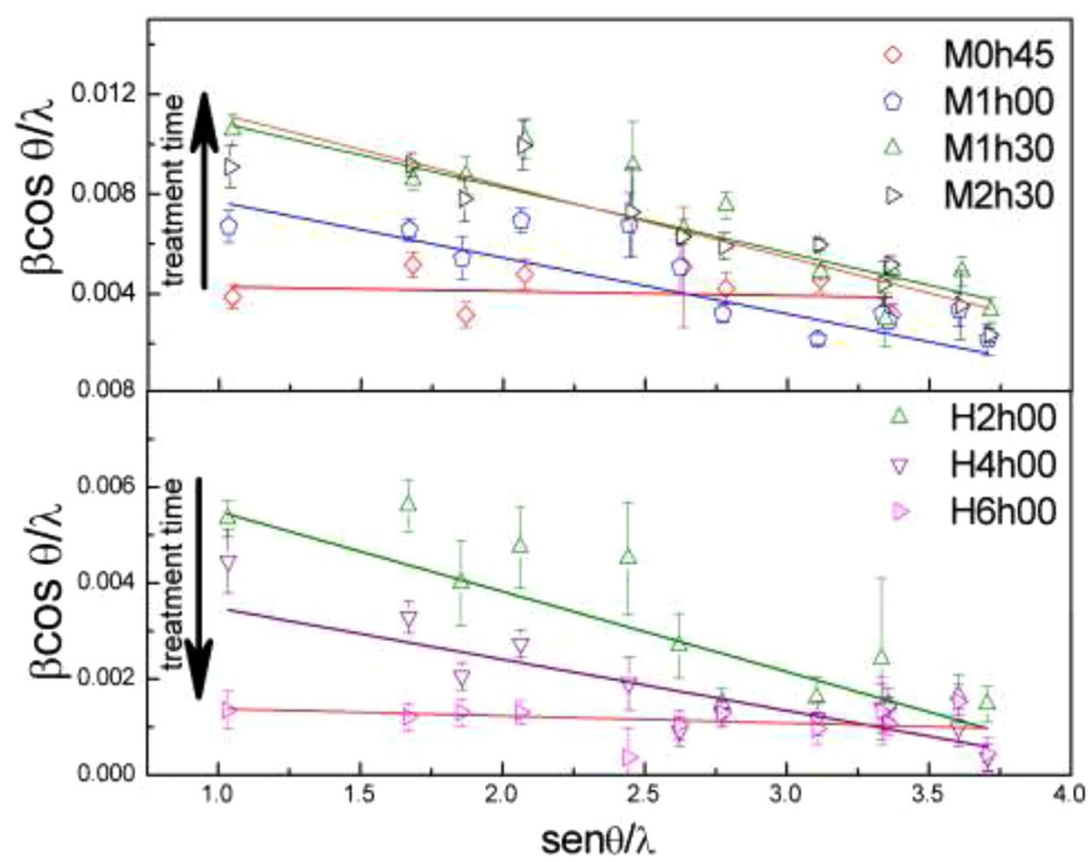

Figure 5. Williansom-Hall plot for SnO nanoparticles, where $\beta$ stands for FWHM (full width half maximum) from the XRD peaks after instrumental correction, $\theta$ is the diffraction angle and, $\lambda$ is the X-ray wavelength. (a) Conventional hydrothermal and, (b) microwave thermal treatment.

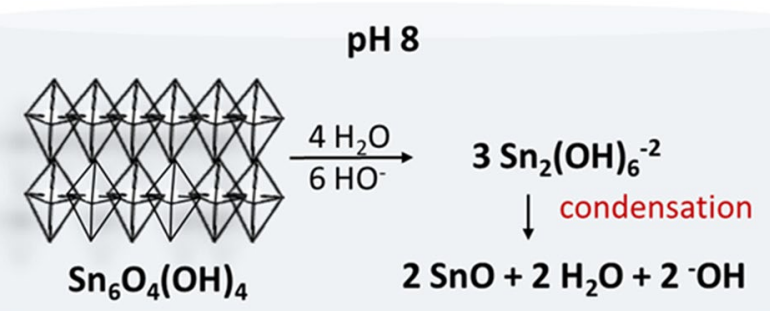

Figure 6. Dissolution-recrystallization steps for the formation of $\mathrm{SnO}$ particles from $\mathrm{Sn}_{6} \mathrm{O}_{4}(\mathrm{OH})_{4}$ intermediate.

\begin{tabular}{|l|l|l|}
\hline Samples & $\mathbf{D}_{\text {WH }}(\mathbf{n m})$ & $\varepsilon(\%)$ \\
\hline H0h45 & - & - \\
\hline H1h00 & - & - \\
\hline H2h00 & $112(16)$ & $-0.0017(3)$ \\
\hline H4h00 & $176(26)$ & $-0.0011(3)$ \\
\hline H6h00 & $523(53)$ & $-0.00015(4)$ \\
\hline M0h45 & $179(19)$ & $\sim 0$ \\
\hline M1h00 & $81(11)$ & $-0.0023(4)$ \\
\hline M1h30 & $59(4)$ & $-0.0026(3)$ \\
\hline M2h30 & $57(6)$ & $-0.0029(4)$ \\
\hline
\end{tabular}

Table 3. Crystallite size $\left(\mathrm{D}_{\mathrm{WH}}\right)$ and strain $(\varepsilon)$ determined via Williamson-Hall Method for the SnO crystal phase.

microwave treatment, the negative strain is related to the shrinkage of the unit cell showed by the Rietveld results (Table 2), which can also be related to the decrease in the nanoparticle size ${ }^{46}$.

Formation of SnO-NPs. Dissolution-recrystallization and oriented-attachment (OA) have been assigned to the formation of $\mathrm{SnO}$ particles with different textures and sizes ${ }^{47}$. In the present case, OA is ruled out because there is no evidence of an assembled architecture. Thus, SnO particles should be build up through dissolution-recrystallization way. In this pathway, the formation of $\mathrm{SnO}$ crystals is derived from the 


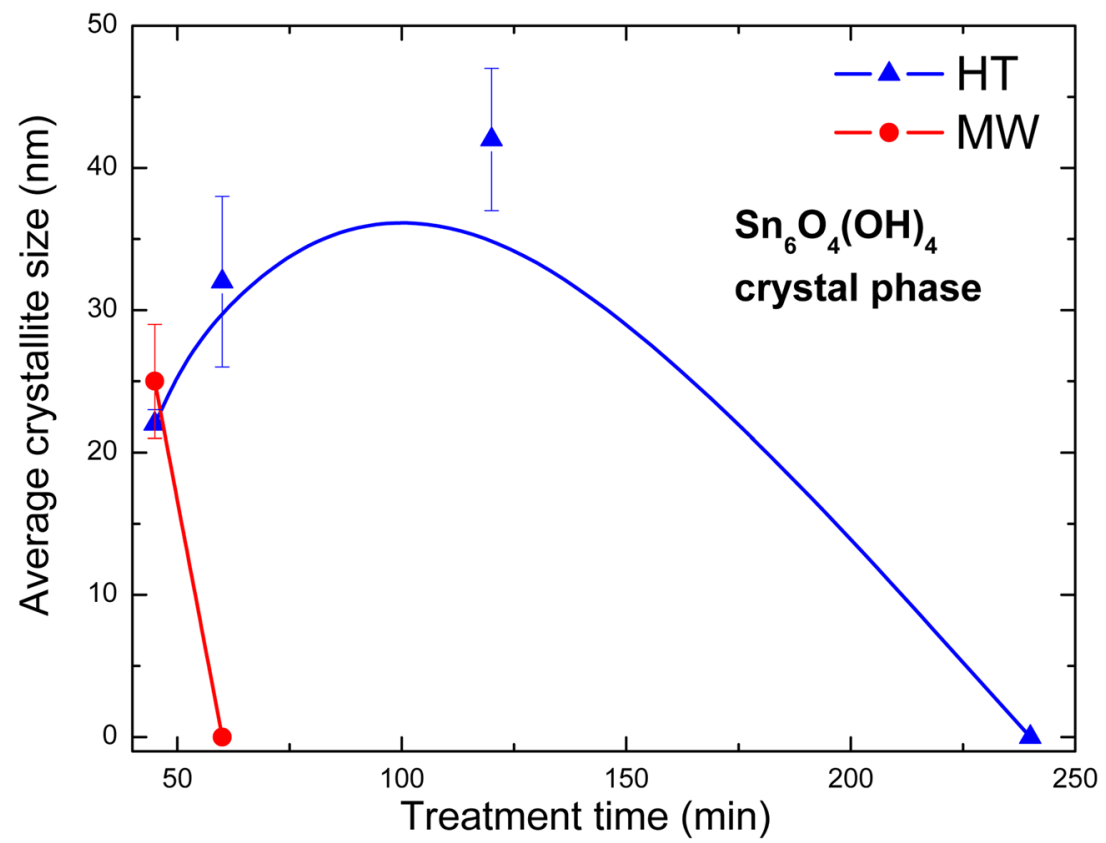

Figure 7. The development of the average crystallite size of the $\mathrm{Sn}_{6} \mathrm{O}_{4}(\mathrm{OH})_{4}$ phase during hydrothermal and microwave treatment time.

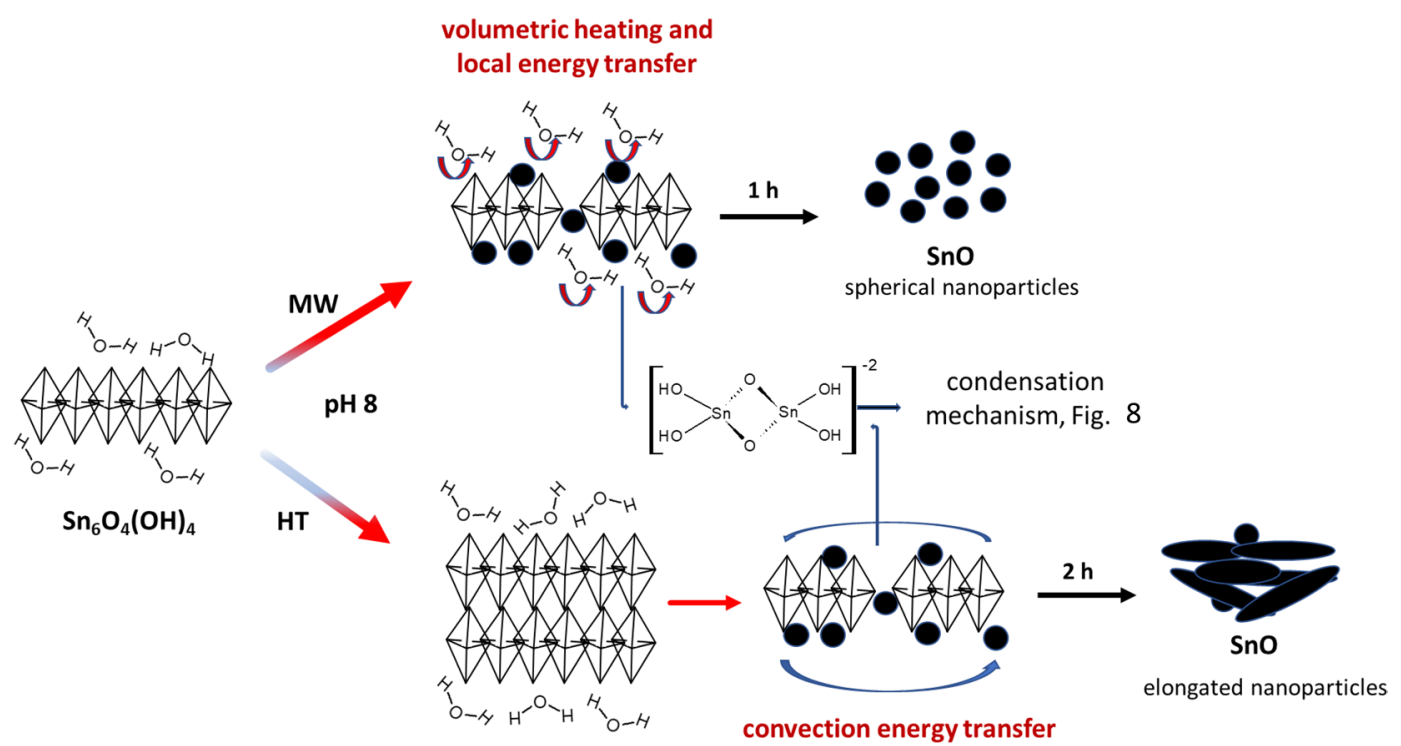

Figure 8. The effect of the conventional and microwave heating in the formation mechanism of SnO-NPs from $\mathrm{Sn}_{6} \mathrm{O}_{4}(\mathrm{OH})_{4}{ }^{53}$.

decomposition of the oxo-hydroxo intermediate, $\mathrm{Sn}_{6} \mathrm{O}_{4}(\mathrm{OH})_{4}$, and then further condensation of the tin-hydroxo complexes showed in Fig. 5, proposed for aqueous-based preparations carried out at $\mathrm{pH} \sim 8$.

Firstly, $\mathrm{Sn}_{6} \mathrm{O}_{4}(\mathrm{OH})_{4}$ is dissolved by the attack of the hydroxide anion, ${ }^{-} \mathrm{OH}$, and water molecules forming the anionic $\mathrm{Sn}_{2}(\mathrm{OH})_{6}{ }^{-2}$ complex, where two $\mathrm{Sn}^{+2}$ and hydroxo groups are bound by bridging, then it condenses to form $\mathrm{SnO}$ particles delivering water and hydroxide anion ${ }^{47}$. In this mechanism, in the overall synthesis, ${ }^{-} \mathrm{OH}$ is consumed.

The synthesis efficiency may also be correlated to the favorable equilibrium in aqueous solution at $\mathrm{pH} \sim 8$. SnO's phase diagram, relating concentration as a function of $\mathrm{pH}$, shows that solubility of the ionic oxo, $\mathrm{HSnO}^{-2}$, and hydroxo, $\mathrm{Sn}(\mathrm{OH})_{2}$, complexes are low $\left(<10^{-6} \mathrm{M}\right)^{30,31}$, thus, the equilibrium is promoted to SnO solid phase. Moreover, species of higher oxidation state can be suppressed, inasmuch as the reaction is taken under an inert atmosphere. Therefore this condition seems to be optimized to grow $\mathrm{SnO}$ particles in spite of the consumption of base throughout the stepwise mechanism. 


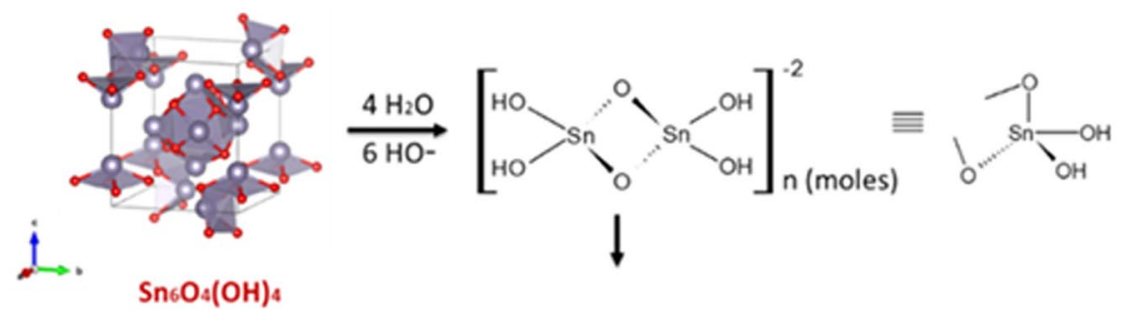

A
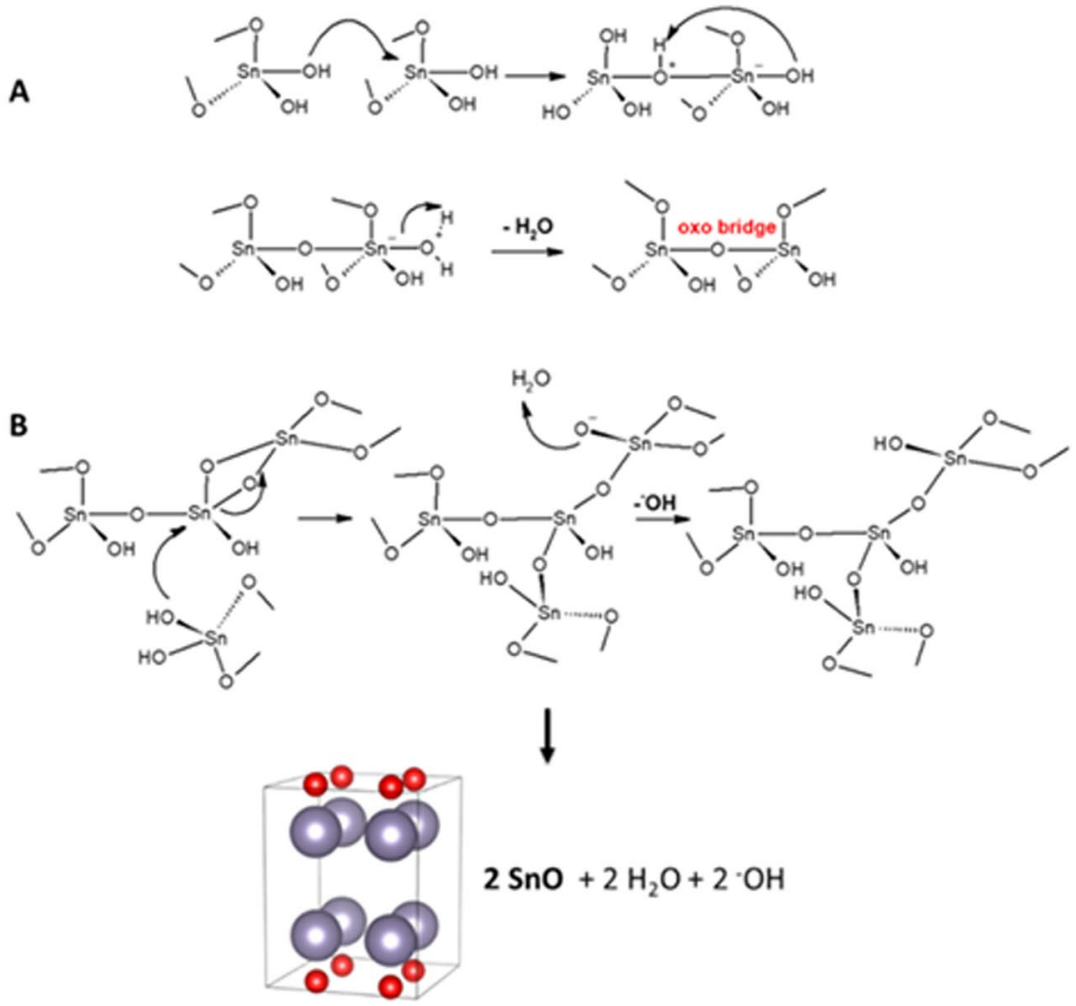

$2 \mathrm{SnO}+2 \mathrm{H}_{2} \mathrm{O}+2 \cdot \mathrm{OH}$

Figure 9. Mechanism proposition of condensation steps: the possible pathways of the dissolution of $\mathrm{Sn}_{6} \mathrm{O}_{4}(\mathrm{OH})_{4}$ and oxolation of the $\mathrm{Sn}_{2}(\mathrm{OH})_{6}{ }^{-2}$ to form SnO-NPs ${ }^{43,44,49-54}$.

Microwave versus Conventional oven heating. The heat-generation from microwave irradiation has been attributed to two main physical processes, namely as dipolar polarization and ionic conduction. Both are accountable for the gains such as decreased reaction rate and selectivity to obtain metal oxides ${ }^{48-50}$. Their magnitudes depend on the bulk dielectric permittivity $(\varepsilon)$, a measure of the extent to which a material can be polarised by an external electric field, that can be expressed as $\varepsilon=\varepsilon^{\prime}+\varepsilon^{\prime \prime} \mathrm{j}$, where $\varepsilon^{\prime}$ is the dielectric constant of the material, $\varepsilon$ " is the dielectric loss and $\mathrm{j}$ is the imaginary number where $\mathrm{j}^{2}=-1^{21}$; the latter term $\varepsilon$ " is related to the gain heating. Laybourn and co-workers have considered the role of the $\varepsilon$ on the synthesis of MOFs under microwave irradiation and elucidate the influence of the concentration, ionic strength as salts are used, temperature, and microwave cavity as well. All these factors have been showed as significant, and even anion- and cation-ligand bond or interaction may exert a fair interference on the heating and reactivity ${ }^{51}$. Herein, microwave heating speeds up the formation of SnO-NPs even at a lower temperature than that for hydrothermal preparation leading to small and spherical-shaped SnO-NPs. Conversely, long particles and sheets morphologies were obtained by the conventional-hydrothermal preparation, in which heating is distributed by convection currents. Hence, one may argue that microwave delivers uniform heat through the reaction medium, which accelerates the rate of nucleation leading to small nanoparticles. On the other hand, the inhomogeneous heat spreading, under hydrothermal reaction, leads to particles of different shapes and sizes and certainly favors the ripening processes.

XRD results (see Figs. 2 and 3) showed that different intervals exist for starting the conversion from $\mathrm{Sn}_{6} \mathrm{O}_{4}(\mathrm{OH})_{4}$ to $\mathrm{SnO}$. Under microwave, it seems to be taking place at earlier reaction time while it happens only after $1 \mathrm{~h}$ through the hydrothermal treatment. This delay-effect has been noticed in another study concerning reaction time dependence to form $\mathrm{SnO}$ crystals $^{49}$. Three possible explanations are $i$. a previous formation of an unknown tin-based structure, after then the condensation happens; $i i$. this interval is needed to increase the concentration of the $\mathrm{Sn}_{2}(\mathrm{OH})_{6}{ }^{-2}$. iii. a unique effect might occur under microwave irradiation. The former may be discarded since that XRD analyses only identified the $\mathrm{Sn}_{6} \mathrm{O}_{4}(\mathrm{OH})_{4}$ and/or SnO phases. Likewise, the second option was dismissed toward the balance of the end up mass obtained that showed practically equal for all reaction: $\mathrm{m}=3.50(3) \mathrm{g}$. If there was a time interval for concentrating the $\mathrm{Sn}_{2}(\mathrm{OH})_{6}{ }^{-2}$ in solution, the final mass of 
solid would decrease in those periods before converting to $\mathrm{SnO}$. Therefore, microwave irradiation should induce a specific effect on reactivity. In order to understand the development of the $\operatorname{Sn}_{6} \mathrm{O}_{4}(\mathrm{OH})_{4}$ crystalline precursor phase, the average crystallite size was measured from the most intense XRD peaks for both microwave (MW) and hydrothermal (HT) synthesis, Fig. 7.

It can be seen that a second-order event in which $\mathrm{HT}$ treatment induces growth of the $\mathrm{Sn}_{6} \mathrm{O}_{4}(\mathrm{OH})_{4}$ crystallite size until $\sim 80 \mathrm{~min}$, then the size decreases accompanying the conversion of $\operatorname{Sn}_{6} \mathrm{O}_{4}(\mathrm{OH})_{4}$ to SnO. On the other hand, in the MW treatment, there is a very quick and straight decrease along with the conversion to SnO. In addition, the WHP analysis (see Fig. 5) showed that HT crystallite tensions decrease just after $2 \mathrm{~h}$ whereas ones from MW crystallite get an early and straight increase. Therefore, assuming a recrystallization-dissolution mechanism in which such an $\mathrm{Sn}_{2}(\mathrm{OH})_{6}{ }^{-2}$ anionic complex is formed, these findings allow to set down the following panorama, shown in Fig. 8: $i$. MW treatment speeds up the formation of the anionic complex up to its saturation, then, the condensation happens inside a uniform heat distribution, favoring faster nucleation and smaller particles; ii. as the HT treatment needs first to achieve an average work temperature, the precursor $\mathrm{Sn}_{6} \mathrm{O}_{4}(\mathrm{OH})_{4}$ grows up in disordered directions until $\sim 1 \mathrm{~h}$, then followed by $\mathrm{SnO}$ particle formation. After two hours, the whole system gathers enough energy to develop the condensation step form $\mathrm{SnO}$ from $\mathrm{Sn}_{2}(\mathrm{OH})_{6}{ }^{-2}$, in spite of the inhomogeneous heat flux.

Moreover, taking into account the interference that microwave assisted-synthesis can have due to the variations of the bulk dielectric permittivity $(e)^{50,51}$, it is not easy to predict its action on the mechanism of condensation, but should be mainly on the steps in which water acts as attacking or living group, i.e., dissolution of $\mathrm{Sn}_{6} \mathrm{O}_{4}(\mathrm{OH})_{4}$ and oxolation of the $\mathrm{Sn}_{2}(\mathrm{OH})_{6}{ }^{-2}$. The oxolation happens through an addition-elimination with a proton-transfer step-mechanism that releases water as leaving group forming an oxo bond, Fig. 9 . As seen in the literature previously, the enhanced maturation of hydroxyapatite under microwave irradiation has been driven by the strong excitation or stretching of the calcium-water bond. Furthermore, the proposed mechanism also provides a path to the final hydroxide, ${ }^{-} \mathrm{OH}$, formation ${ }^{52}$. In our case such an effect should take place on the stepwise in which water is withdrawn, accelerating $\mathrm{SnO}$ formation The tetrahedra complex $\mathrm{Sn}_{2}(\mathrm{OH})_{6}{ }^{-2}$ has two oxo bridges that are attacked by the hydroxo nucleophilic group via a nucleophilic substitution, further opening a cycle, producing the tin-alkoxy, $\mathrm{Sn}-\mathrm{O}^{-}$, that takes off a proton from water delivering ${ }^{-} \mathrm{OH}$.

\section{Conclusion}

Conventional- and microwave-hydrothermal aqueous syntheses to form $\mathrm{SnO}$ were carried out at $\mathrm{pH} 8$ under an inert atmosphere. The efficiency of conversion from $\mathrm{Sn}_{6} \mathrm{O}_{4}(\mathrm{OH})_{4}$ to $\mathrm{SnO}$ was verified in the different reaction times by XRD analysis, as well as the structural and morphological aspects of the particles. Conventional hydrothermal treatment yielded $\mathrm{SnO}$ sub-micrometric particles with sizes varying from 30 to $550 \mathrm{~nm}$ after 4 hours. By the microwave route, uniformly and spherical-shaped $\mathrm{ZnO}$-NPs with $4 \mathrm{~nm}$ average size were obtained after 2 hours. XRD analyses indicate that size and morphology have a treatment time dependence. The XRD results were different from those observed by TEM, which can be attributed to samples' preparation, leading to larger agglomerates. The mechanism of the formation of $\mathrm{SnO}$ may be assigned to a dissolution-recrystallization process, and the acceleration provided by the microwave-assisted preparation may be attributed to a better heat distribution into the synthesis medium.

Received: 3 January 2020; Accepted: 13 May 2020;

Published online: 10 June 2020

\section{References}

1. Allen, J. P., Scanlon, D. O., Parker, S. C. \& Watson, G. W. Tin monoxide: structural prediction from first-principles calculations with van der Waals corrections. The Journal of Physical Chemistry C 115(40), 19916-19924 (2011).

2. Wang, H. \& Rogach, A. L. Hierarchical SnO2 nanostructures: recent advances in design, synthesis, and applications. Chemistry of Materials 26(1), 123-133 (2013).

3. Jiang, E. et al. Size-controllable synthesis of monodispersed $\mathrm{SnO} 2$ nanoparticles and application in electrocatalysts. The. Journal of Physical Chemistry B 109(18), 8774-8778 (2005).

4. Li, F. et al. One-step synthesis of graphene/SnO2 nanocomposites and its application in electrochemical supercapacitors. Nanotechnology 20(45), 455602 (2009).

5. Reddy, M. V., Subba Rao, G. V. \& Chowdari, B. V. R. Metal oxides and oxysalts as anode materials for Li-ion batteries. Chemical Reviews 113(7), 5364-5457 (2013)

6. Li, X. et al. Determination of some basic physical parameters of SnO based on SnO/Si PN heterojunctions. Applied Physics Letters 106(13), 132102 (2015).

7. Minami, T. Substitution of transparent conducting oxide thin films for indium tin oxide transparent electrode applications. Thin solid films 516(7), 1314-1321 (2008).

8. Martinez, C. J., B. Hockey, C. B., Montgomery, S. \& Semancik Porous tin oxide nanostructured microspheres for sensor applications. Langmuir 21(17), 7937-7944 (2005).

9. Saji, K. J., Subbaiah, Y. V., Tian, K. \& Tiwari, A. P-type SnO thin films and SnO/ZnO heterostructures for all-oxide electronic and optoelectronic device applications. Thin Solid Films 605, 193-201 (2016).

10. Ogo, Y. et al. Tin monoxide as an s-orbital-based p-type oxide semiconductor: Electronic structures and TFT application. Physica status solidi (a) 206(9), 2187-2191 (2009).

11. Nomura, K., Kamiya, T., H. \& Hosono Ambipolar oxide thin-film transistor. Advanced Materials 23(30), 3431-3434 (2011).

12. Calderer, J. et al. Synthesis and characterization of metal suboxides for gas sensors. Microelectronics Reliability 40(4-5), 807-810 (2000).

13. Cherian, C. T., Reddy, M. V., Haur, S. C., B.V.R. \& Chowdari Facile synthesis and Li-storage performance of SnO nanoparticles and microcrystals. RSC Advances 3(9), 3118-3123 (2013).

14. Ortiz, G. F. et al. Nanoarchitectured TiO2/SnO: a future negative electrode for high power density Li-ion microbatteries? Chemistry of Materials 22(5), 1926-1932 (2010).

15. Wang, L. et al. Large-scale preparation of shape-controlled $\mathrm{SnO}$ and improved capacitance for supercapacitors: from nanoclusters to square microplates. Nanoscale 5(16), 7613-7621 (2013). 
16. Kim, A. Y. et al. Ordered SnO nanoparticles in MWCNT as a functional host material for high-rate lithium-sulfur battery cathode. Nano. Research 10(6), 2083-2095 (2017).

17. Zhang, J. et al. Electrical transport properties of SnO under high pressure. The. Journal of Physical Chemistry C 115(42), 20710-20715 (2011).

18. Wang, J. et al. One-pot hydrothermal synthesis of highly efficient $\mathrm{SnOx} / \mathrm{Zn} 2 \mathrm{SnO} 4$ composite photocatalyst for the degradation of methyl orange and gaseous benzene. Applied Catalysis B: Environmental 200, 19-30 (2017).

19. Safaei-Ghomi, J., Shahbazi-Alavi, H., E. \& Heidari-Baghbahadorani SnO nanoparticles as an efficient catalyst for the one-pot synthesis of chromeno[2,3-b]pyridines and 2-amino-3, 5-dicyano-6-sulfanyl pyridines. RSC Advances 4(92), 50668-50677 (2014).

20. Sun, G. et al. Hydrothermal synthesis of honeycomb-like SnO hierarchical microstructures assembled with nanosheets. Materials Letters 98, 234-237 (2013).

21. J. Gajendiran, V. \& Rajendran. A study of the nano-structured aggregated tin oxides $(\mathrm{SnO} 2 / \mathrm{SnO})$ and their structural and photoluminescence properties by a hydrothermal method. Materials Letters, 139, 116-118 (2015).

22. Cui, Y. et al. Synthesis of novel 3D SnO flower-like hierarchical architectures self-assembled by nano-leaves and its photocatalysis. Materials Research Bulletin 70, 784-788 (2015).

23. Cheng, H. et al. Electrochemical fabrication and optical properties of porous tin oxide films with structural colors. Journal of Applied Physics 116(15), 153511 (2014).

24. Zheng, H., Gu, C. D., Wang, X. L. \& Tu, J. P. Fast synthesis and optical property of SnO nanoparticles from choline chloride-based ionic liquid. Journal of nanoparticle research 16(2), 2288 (2014).

25. Aurbach, D. et al. Nanoparticles of $\mathrm{SnO}$ produced by sonochemistry as anode materials for rechargeable lithium batteries. Chemistry of Materials 14(10), 4155-4163 (2002).

26. Nguyen, M. T. et al. Reproducible shape control of single-crystal SnO microparticles. RSC Advances 6(32), 26725-26733 (2016).

27. Zima, T., I. \& Bataev Morphology and phase transformations of tin oxide nanostructures synthesized by the hydrothermal method in the presence of dicarboxylic acids. Journal of Solid State Chemistry 243, 282-289 (2016).

28. Wang, S. et al. Solution route to single-crystalline SnO platelets with tunable shapes. Chemical Communications 4, 507-509 (2005).

29. Guo, Y. Q. et al. Shape-controlled growth and single-crystal XRD study of submillimeter-sized single crystals of SnO. CrystEngComm 13(19), 5677-5680 (2011).

30. Sakaushi, K. et al. Synthesis and applications of SnO nanosheets: parallel control of oxidation state and nanostructure through an aqueous solution route. Small 6(6), 776-781 (2010).

31. Uchiyama, H., Ohgi, H., H. \& Imai Selective preparation of $\mathrm{SnO} 2$ and $\mathrm{SnO}$ crystals with controlled morphologies in an aqueous solution system. Crystal growth \& design 6(9), 2186-2190 (2006).

32. R. A. Young The Rietveld Method (International Union of Crystallography Monographs on Crystal). Oxford University Press, USA, (1995).

33. Rietveld, H. M. Line profiles of neutron powder-diffraction peaks for structure refinement. Acta Crystallographica 22(no. 1), 151-152 (1967)

34. Rietveld, H. M. A profile refinement method for nuclear and magnetic structures,. Journal of Applied Crystallography 2(no. 2), 65-71 (1969).

35. McCusker, L. B. et al. Rietveld refinement guidelines. Journal of Applied Crystallography 32, 36-50 (1999).

36. J. Rodriguez-Carbajal, Fullprof Suite; version 3.5d. Institute Laue-Langevin, Grenoble, France, 2009-2012.

37. J. Rodriguez-Carvajal, An introduction to the program Fullprof 2000. Laboratoire Leon Brillouin (CEA-CNRS), (2001).

38. T. Degen, M. Sadki, E. Bron, U. König, G. Nénert The HighScore suite, Powder Diffraction, 29 S2, S13-S18 (2014).

39. Williamson, G. \& Hall, W. X-ray line broadening from filed aluminum and wolfram. Acta Metallurgica 1(1), 22-31 (1953).

40. R. Guinebretière, X-ray Diffraction by Polycrystalline Materials, ISTE, London, (2007).

41. Langford, J. I. \& Wilson, A. J. C. Scherrer after sixty years: a survey and some new results in the determination of crystallite size. Journal of applied crystallography 11, 102-113 (1978)

42. C. A. Schneider, W. S. Rasband, K.W. Eliceiri. NIH Image to ImageJ: 25 years of image analysis. Nature methods 9, 671-675.

43. I. Abrahams, S.M. Grimes, S.R. Johnston, J.C. Knowles (1996). Acta Crystallographica, Sec. C, 52286 (2012).

44. Izumi, F. J. Solid State Chem. 38381 (1981).

45. Brazuna, L. P. et al. Effect of Lithium and Sodium ions on size and morphology of $\mathrm{ZnO}$ nanoparticles synthesized by glycerol-urea route. New J. Chem. 43, 18988-18995 (2019).

46. Langford, J. I., Cernik, R. J. \& Louër, D. The Breadth and Shape of Instrumental Line Profiles in High-Resolution Powder. Diffraction. J. Appl. Cryst. 24, 913-919 (1991).

47. Zhang, R. et al. Morphology modulation of $\mathrm{SnO}$ photocatalyst: from microplate to hierarchical architectures self-assembled with thickness controllable nanosheets. CrystEngComm 20(32), 4651-4665 (2018).

48. Bilecka, I. \& Niederberger, M. Microwave chemistry for inorganic nanomaterials synthesis. Nanoscale 2(8), 1358-1374 (2010).

49. El-Rafei, A. M., Youssef, H. \& Ahmed, N. M. Synthesis and Characterization of Nano-/Micro-Crystalline SnO using Microwave and Hydrothermal Techniques. Journal of Ceramic Science and Technology 5(3), 217-222 (2014).

50. Laybourn, A. et al. Metal-organic frameworks in seconds via selective microwave heating. Journal of Materials Chemistry A 5(16), 7333-7338 (2017).

51. Laybourn, A. et al. Understanding the electromagnetic interaction of the metal-organic framework reactants in aqueous solution at microwave frequencies. Physical Chemistry Chemical Physics 18(7), 5419-5431 (2016).

52. Lerner, E., Sarig, S. \& Azoury, R. Enhanced maturation of hydroxyapatite from aqueous solutions using microwave irradiation. $J$ Mater Sci: Mater Med 2, 138-141 (1991).

53. Jolivet, J.-P., Chanéac, C. \& Tronc, E. Iron oxide chemistry. From molecular clusters to extended solid networks. Chem. Commun. 5, $477-483$ (2004).

54. Momma, K. \& Izumi, F. VESTA 3 for three-dimensional visualization of crystal, volumetric and morphology data. J. Appl. Crystallogr. 44, 1272-1276 (2011).

\section{Acknowledgements}

We would like to thank the FAPESP funding agency (grant numbers 2016/17708-4, 2017/13839-0 and, 2013/08166-5. We also thank Prof. Mario Politi for the discussions.

\section{Author contributions}

Juliana Silva Dias made the synthesis and characterization of all samples as part of her undergraduate science project and prepared the references. Fabio Rodolfo Miguel Batista helped with the discussion about microwave effect. Rebeca Bacani made and wrote the structural and morphological characterization, and prepared Tables (1-3) and Figs. (2-5, 7). Eduardo Rezende Triboni wrote the main manuscript text and prepared Figs. (6, 8 and 9).

\section{Competing interests}

The authors declare no competing interests. 


\section{Additional information}

Correspondence and requests for materials should be addressed to E.R.T.

Reprints and permissions information is available at www.nature.com/reprints.

Publisher's note Springer Nature remains neutral with regard to jurisdictional claims in published maps and institutional affiliations.

(c) (i) Open Access This article is licensed under a Creative Commons Attribution 4.0 International License, which permits use, sharing, adaptation, distribution and reproduction in any medium or format, as long as you give appropriate credit to the original author(s) and the source, provide a link to the Creative Commons license, and indicate if changes were made. The images or other third party material in this article are included in the article's Creative Commons license, unless indicated otherwise in a credit line to the material. If material is not included in the article's Creative Commons license and your intended use is not permitted by statutory regulation or exceeds the permitted use, you will need to obtain permission directly from the copyright holder. To view a copy of this license, visit http://creativecommons.org/licenses/by/4.0/.

(C) The Author(s) 2020 